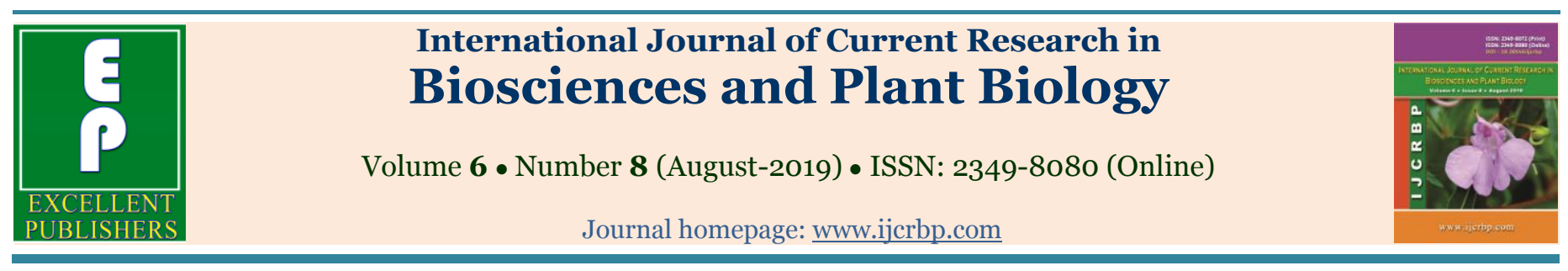

\title{
Screening of PHB biopolymer producing Bacillus cereus from municipal sludge waste
}

\author{
P. Murali, K. Mamatha and N. Mathiyazhagan* \\ PG and Research Centre in Biotechnology, MGR College, Hosur- 635 130, Krishnagiri, Tamil Nadu, India \\ *Corresponding author; e-mail: mathimicro@gmail.com
}

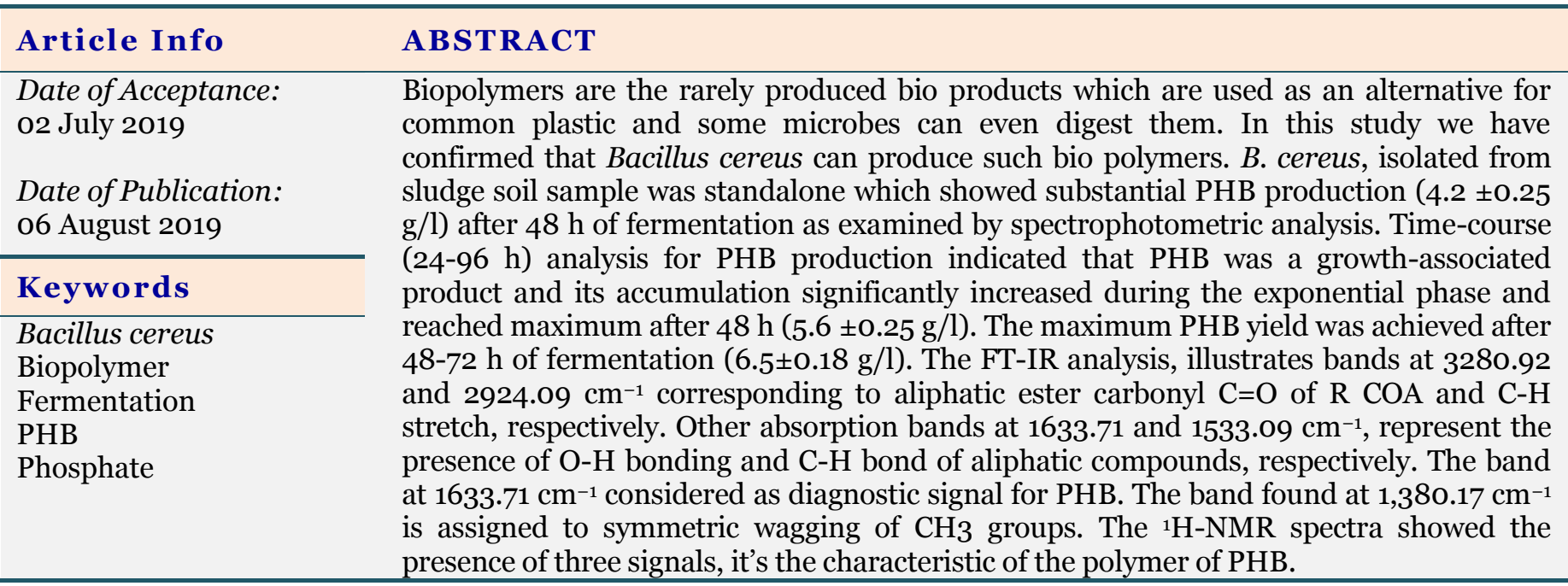

\section{Introduction}

Plastics are a ubiquitous part of the modern life, and their molecular structure can be manipulated to meet the requirements of almost any imaginable application including construction, health care, transportation, food packaging, consumer goods and communications. Currently the vast majority of plastics in use are manufactured using a petrochemical route, derived from materials of fossil oil and gas origin. About 4 to $6 \%$ of the world's annual petroleum production is used for the production of plastics and more $4 \%$ is utilised to power manufacturing processes (British Plastics Federation, 2017).
Plastics are extremely versatile materials and have been essential in the development of society since the 2oth century, though they have environmental impact associated not only with their manufacture, but also at their disposal. This is because plastics have a slow degradation rate, making them environmentally persistent and hence presenting a serious pollution issue in land and oceans (Marshall et al., 2013). These alternative types of plastic must retain the desired properties of traditional synthetic plastics and, ideally, should biodegrade completely without leaving any undesirable remains once discarded. Biopolymers can be mainly classified into four groups based on their biodegradable characteristics (Mudgal et al., 2013). 
The properties of Polyhydroxybutyrate (PHB) are similar to many synthetic thermoplastic polymers, such as polypropylene, making PHB a promising candidate as a drop in replacement for petroleumbased plastics in wide range of applications such as paper coatings, packaging and manufacturing of plates and bottles (Batcha et al., 2014). Previous research on PHB has focused on making production economical and improving the quality of biopolymers such that they are able to compete with the physical and mechanical properties of petrochemical- derived polymers, allowing biopolymers to be utilised as direct replacements for commonly used synthetic polymers (Batcha et al., 2014).

More than 200 types of microorganisms are able to accumulate PHB, such as photosynthetic bacteria, archaebacteria, and Gram negative and Grampositive bacteria. B. cereus is a Gram-positive bacterium that is a model PHB producing microorganism because it is able to accumulate PHB to a high level of $90 \%$ of dry cell weight (Chee et al., 2015). Unfortunately, B. cereus can grow on glucose, the main sugar that is obtained from cellulosic material, which limits the use of this strain for PHB production using sources of nonedible, lingocellulosic biomass. There is potential to use for sludge soil.

\section{Materials and methods}

\section{Collection and processing of soil sample}

The soil sample was collected from the lake (sludge soil) of Hosur, Krishnagiri district of Tamil Nadu. Collected samples were immediately processed and stored in refrigerator for further process in the PG and Research Centre in Biotechnology of MGR College, Hosur.

\section{Isolation and screening of $\mathrm{PHB}$ producing Bacillus cereus}

Isolation of PHB producing bacteria was accomplished based on the methods of Khanna and Srivastava (2005) with slight modifications. The microbes were isolated from collected sludge soil sample by standard serial dilution techniques by using saline water. $10^{5}$ and $10^{6}$ dilutions were taken on selective and standard media for screening of PHB producing bacteria. Briefly the dilutions were inoculated $5 \% \mathrm{w} / \mathrm{v}$ on selective media such as Bacillus cereus selective agar base enriched with Disodium hydrogen phosphate (2.5g) and Pottasium dihydrogen phosphate (0.25g), Peptone (1g), Mannitol (10g), Sodium chloride (2g), Megnesium sulphate (o.1g), Sodium pyruvate (10g), Bromothymol blue (0.12g) and agar (2g) and incubated at $30^{\circ} \mathrm{C}$ for $24 \mathrm{~h}$ in an shaker incubator. Plates were incubated at $30^{\circ} \mathrm{C}$ for 2-3 days, and colonies appeared and was exposed to visible light and those showing slight bluish colonies were earmarked as presumptive PHB producers (Thammasittirong et al., 2017).

PHB producing ability of bacterial isolates was examined further by confocal microscopic analysis by using Sudan black, a lipophilic dye (Model Olympus Fluoview Ver 1-7b) as described by Hermawan and Jendrossek (2007). The suspected PHB producing bacterial isolates were first grown in PHB production medium (PPM) which consisted of $\left(\mathrm{g} / \mathrm{l}^{-1}\right): \mathrm{MgSO}_{4} 7 \mathrm{H} 2 \mathrm{O}$ o.1, $\mathrm{K}_{2} \mathrm{HPO} 4$ $0.5, \mathrm{NH}_{4} \mathrm{NO}_{2}$ 0.1, glucose 20.0, malt extract 0.5 , yeast extract 1.0, $\mathrm{pH} 7$, for $48 \mathrm{~h}$. Cells were harvested, and immediately stained with $0.1-0.5$ volumes of Sudan black solution (0.1-1 mg ml-1 in ethanol). The dark bluish colour results conformed the PHB producing bacteria isolates.

\section{Characterization of selected isolate}

Among the bacterial isolates screened for PHB production, the one which generated maximum PHB was selected, and examined based on morphological, physiological, biochemical properties and staining techniques. Biochemical analysis included carbohydrate fermentation tests, Indole, methyl red and Voges-Proskauer test (IMVIC), catalase, Glucose peptone agar (GPA) test, Gelatin test. The conformed bacterial isolates were maintained by frequent sub culturing process. Among various bacterial isolated $B$. cereus was taken for further study.

\section{Production of PHB}

All the PHB producing isolates were subjected to submerged fermentation for PHB production. Inoculum was developed by cultivating the cells in production medium under shaking at $30^{\circ} \mathrm{C}$ for $18 \mathrm{~h}$ to attain absorbance (A600) of 0.8-0.9 (approx. $\left.10^{8} \mathrm{cfu} / \mathrm{ml}\right)$ and inoculated at $5 \%(\mathrm{v} / \mathrm{v})$ for PHB 
production. Fermentation was executed at $300 \mathrm{C}$ under shaking at $150 \mathrm{rpm}$. Fermented broth was centrifuged (Eppendorf Centrifuge 5804-R, Germany) at different time intervals (24-96 h) and the resultant biomass was used for PHB extraction (Khanna and Srivastava, 2005).

\section{Extraction and quantification of PHB production}

All the Sudan Black B positives isolates were subjected to quantification of PHB production as per the method of Schlegal et. al (1961). The bacterial cells containing the polymer were centrifuged at $10,000 \mathrm{rpm}$ for $10 \mathrm{~min}$ and the pellet was washed with equal volume of acetone and ethanol to remove unwanted materials. The pellet was resuspended into $4 \%$ of sodium hypochlorite and incubated at room temperature for $30 \mathrm{~min}$. The whole mixture was again centrifuged and the supernatant was discarded. The cell pellet containing PHB was again washed with equal volume of acetone and ethanol. Finally the pellet containing the polymer granules were dissolved in hot chloroform (Arshad et al., 2007). The chloroform was filtered, and to the filtrate $10 \mathrm{ml}$ of concentrated hot sulphuric acid was added. The addition of sulphuric acid converts the pellet into crotonic acid which is brown in color. The solution was cooled and the absorbance was read at $400 \mathrm{~nm}$ against sulfuric acid as blank.

\section{Polymer analysis}

\section{Fourier transform-infrared spectroscopy (FT-IR) and NMR analysis}

PHB production from $B$. cereus was executed under submerged fermentation and PHB produced was subjected for IR spectra recording in the range $4000-400 \mathrm{~cm}^{-1}$ at a resolution of $4 \mathrm{~cm}^{-1}$ to confirm the functional groups of the extracted polymer as per the method described by Liau et al. (2014). All the experiments were performed in triplicates and all the data is represented here is the Mean +/- SD.

NMR Analysis: ${ }^{1} \mathrm{H}$ Nuclear Magnetic Resonance

The identity of individual monomer unit was confirmed by proton nuclear magnetic resonance ( $\left.{ }^{1} \mathrm{H}-\mathrm{NMR}\right)$ spectroscopy. ${ }^{1} \mathrm{H}-\mathrm{NMR}$ spectra of $\mathrm{PHB}$ sample were recorded in $\mathrm{CDCl}_{3}$ on Bruker $\mathrm{ACF}$
300 spectrophotometer at $300 \mathrm{MHz}$ by using "Tetramethylsilane" as internal standard.

\section{Results and discussion}

The collected samples were serially diluted and cultured by spread plate method with the following dilutions10 $10^{-3}, 10^{-4}, 10^{-5}, 10^{-6}$ with one control. More than 10 bacterial cultures were isolated from lake (sludge soil) of Hosur, Krishnagiri district of Tamil Nadu obtained on nutrient agar medium. The simplest first line screening program for PHB producing bacteria is the use of Sudan black B staining. The isolates which were found positive for PHB granules after Staining with Sudan black B (i.e., showing dark spot inside the pink coloured cells) were further confirmed for their PHB producing potential with specific media, a more specific dye for PHB granules, in which dark blue colour was observed under the microscopy (Fig. 2 and Fig. 3). The PHB produced bacterial culture was further identified by following experimental results.

\section{Isolation of Bacillus cereus on selective media}

The selective media were prepared and streaked with the suspected colonies in separate plates for each. The isolates grown in only few selective media plates (Bacillus cereus selective agar base) and which are confirmed as Bacillus cereus (Fig. 1).

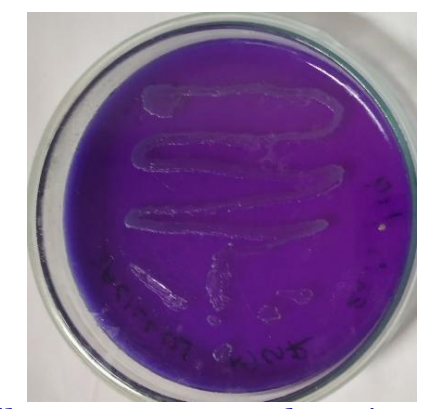

Fig. 1: Bacillus cereus pure culture in selective media.

\section{Conformation of PHB producing Bacillus cereus by Sudan Black -B staining}

The bacteria were initially screened for the PHB production in nutrient broth and the ability to synthesize PHB granules was confirmed using Sudan black. Pure culture of isolated strain was 
done by plating method. Presence of PHB granules was identified and confirmed by Sudan Black- B staining and the granules were observed. The isolates were tested for PHB production following the viable colony screening method based on the intensity of staining. The isolates gave the positive result for PHB accumulation through Sudan black staining method scoring. The Bacillus cereus was treated with Sudan Black- B positive isolates were subjected to quantitative estimation of PHB production. Black color colonies were taken as positive result.

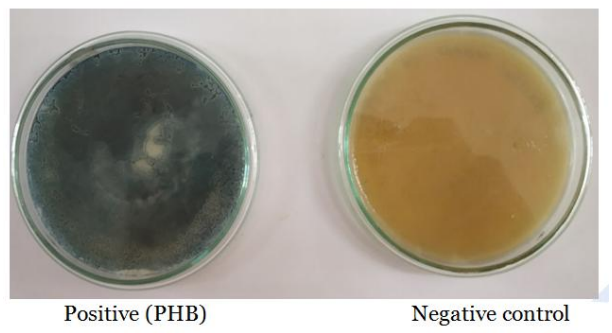

Fig. 2: Sudan Black test.

PHB producing bacterial colonies were showed bluish black color colonies were recorded on Sudan black containing medium. Confocal microscopic analysis of PHB producing bacterial cells indicated appearance of cells filled with red color PHB granules. Alternate staining methods like direct staining of colonies with Sudan black have been used by some researchers for detection of PHB granules (Singh, et al., 2011).

\section{Macroscopic, microscopic and biochemical bacterial analysis and conformation}

The isolates were confirmed by macroscopic and microscopic observations. Therefore, obviously bacterial isolate was selected for further studies including its identification by morphological and biochemical means. Macroscopic analysis showed that bacterial colonies were sticky, discrete and creamy white. Microscopic examination established that bacteria were Gram-positive, rod shaped and sporulating (Fig. 2). Besides, isolate was catalase and methyl red positive but negative for VP test, and hydrolysed casein, starch and lipids. PHB from Gram-positive bacteria may be promising for biomedical and tissue engineering applications (Shivakumar, 2012) as Gram-positive bacteria are devoid of lipopolysaccharides and there is no chance of lipopolysaccharides contamination (Fig. 3).

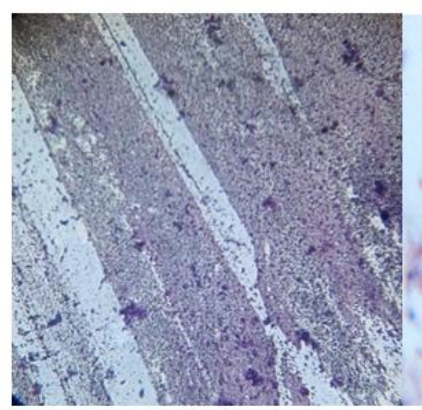

(A)

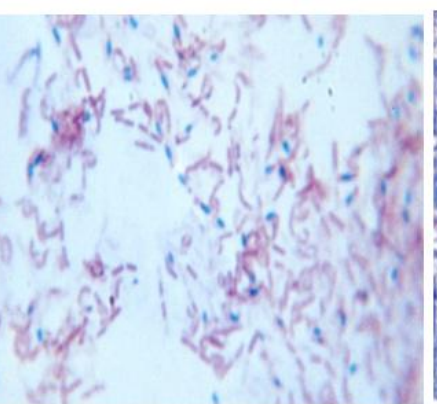

(B)

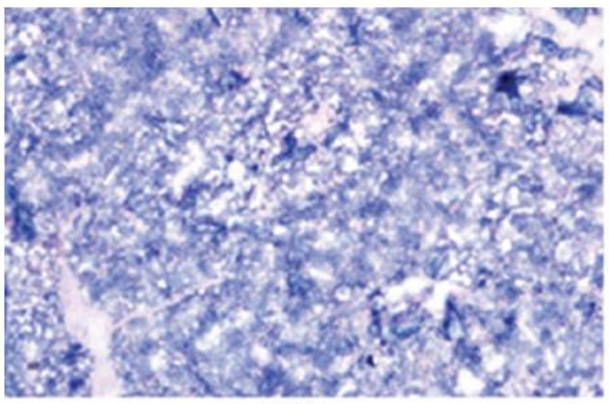

(C)

Fig. 3: Staining results. (A) Gram staining; (B) Endospore staining; (C) Sudan black B staining.

\section{Biochemical characterization of Bacillus isolates}

Biochemical characteristics of isolate was studied on the support of different biochemical test like Glucose Peptone agar assay, and Citrate utilization test, indole test, etc. The cultures were confirmed as Bacillus cereus by the standard biochemical result.

\section{Production of PHB}

After initial screening, $B$. cereus isolate was subjected to submerged fermentation in the PHB production medium. $B$. cereus, isolated from sludge soil sample was standalone which showed substantial PHB production (4.2 g/l \pm 0.25$)$ after $48 \mathrm{~h}$ of fermentation as examined by spectrophotometric analysis. Time-course (24-96 h) analysis for PHB production indicated that PHB was a growth-associated product and its accumulation significantly increased during the exponential phase and reached maximum after 48 $\mathrm{h}(5.6 \pm 0.25 \mathrm{~g} / \mathrm{l})$. The maximum PHB yield was achieved after $48-72 \mathrm{~h}$ of fermentation $(6.5 \pm 0.18$ 
$\mathrm{g} / \mathrm{l})$. After $72 \mathrm{~h}$, a decrease in the level of cell dry weight coincided with a small decrease in PHB content.

During the adverse conditions i.e., with glucose the PHB was used by the cell as an internal reserve of carbon and energy. Furthermore, increase in viscosity of the medium may also lead to unfavorable conditions such as production of extracellular metabolites, presence of an intracellular PHB depolymerase, depletion of essential nutrients in the medium or selfutilization of PHB by bacteria due to nutrient depletion and cells, consumption of PHB as a carbon source (Madison and Huisman, 1999; Ramadas et al., 2009). Fermentation time for maximum PHB production varies among different bacteria and depends largely upon cultural/environmental conditions employed during fermentation and genetic make-up of the organism.

It is desirable that PHB production be attempted by using inexpensive agricultural or other residues as carbon source to substantially reduce the cost of PHB production considering carbon source as the major cost determining factor for PHB production (Valappil et al., 2007). PHB production has been reported from several bacteria by using waste materials or agricultural residues as inexpensive carbon sources (Van-Thuoc et al., 2008; Yezza et al., 2006). Furthermore, readily available lowprice carbon sources may have high amounts of nutrients, such as amino acids and peptides, which contributes to improved cell growth and metabolite biosynthesis and pave the way for resourceful and cost-effective production of PHB. PHB being the reserved food polymer produced during time of starvation is degraded to provide carbon and energy when external carbon source is exhausted (Shivakumar, 2012). However, B. megaterium showed maximum PHB accumulation when glucose was used as carbon source (Hori et al., 2002). Bacillus species has been reported to accumulate PHB up to $90 \%$ of cell dry weight by utilizing soy molasses oligosaccharides like raffinose without the need for nutrient limitation (Full et al., 2006; Das et al., 2017).

Polymer breakdown, so that the PHB is utilized at the rate almost equal to the rate of its synthesis (Singh et al., 2011). Bacillus sphaericus NCIM 5149 showed pH 7.5 as the most apt for PHB production (Ramdas et al., 2009) while for Bacillus species $\mathrm{pH}$ of 6-7 the most effective for PHB accumulation (Singh et al., 2011). Current study showed that inoculum at $5 \%,(\mathrm{v} / \mathrm{v})$ was best for maximum PHB yield $(5.6 \pm 0.15 \mathrm{~g} / \mathrm{l})$.

\section{Extraction and purification of PHB}

After the 3omins incubation of isolates in ice cold methanol, a slimey layer of PHB (polyhydroxybyturate) were observed, and the layer were collected separetely in to a watch glass and dried at $60^{\circ} \mathrm{C}$ for $12 \mathrm{~h}$ (Fig. 4).

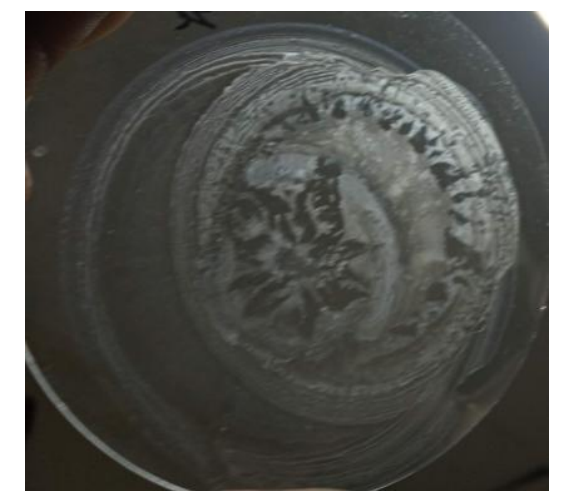

Fig. 4: Watch glass with dried PHB.

Higher or lower inoculum level resulted in yield reduction of $\mathrm{PHB}$ from $B$. cereus IR spectrum of the extracted polymer, illustrates bands at 3280.92 and $2924.09 \mathrm{~cm}^{-1}$ corresponding to aliphatic ester carbonyl $\mathrm{C}=\mathrm{O}$ of $\mathrm{RCOA}$ and $\mathrm{C}-\mathrm{H}$ stretch, respectively. Other absorption bands at 1633.71 and $1533.09 \mathrm{~cm}^{-1}$, represent the presence of $\mathrm{O}-\mathrm{H}$ bonding and $\mathrm{C}-\mathrm{H}$ bond of aliphatic compounds, respectively (Fig. 5). The band at $1633.71 \mathrm{~cm}^{-1}$ considered as diagnostic signal for PHB. The band found at $1,380.17 \mathrm{~cm}^{-1}$ is assigned to symmetric wagging of $\mathrm{CH}_{3}$ groups. The remaining bands located at $1000 \sim 1300 \mathrm{~cm}^{-1}$ correspond to the stretching of the $\mathrm{C}-\mathrm{O}$ bond of the ester group. The results described above are congruent with the findings reported previously. The most prominent marker (ester carbonyl) band for PHB was at 1740 $\mathrm{cm}^{-1}$ and $1724.03 \mathrm{~cm}^{-1}$ for pure PHB (Panda et al., 2010). In pure PHB granule, asymmetrical deformation of $\mathrm{C}-\mathrm{H}$ bond in $\mathrm{CH}_{2}$ groups and $\mathrm{CH}_{3}$ groups $\mathrm{C}=\mathrm{O}$ bond stretching and $\mathrm{C}-\mathrm{O}$ ester bond are represented by wave numbers 1460, 1379, 1726 and $1150 \mathrm{~cm}-1$, respectively (Tripathi et al., 2013; Panda et al., 2018). 


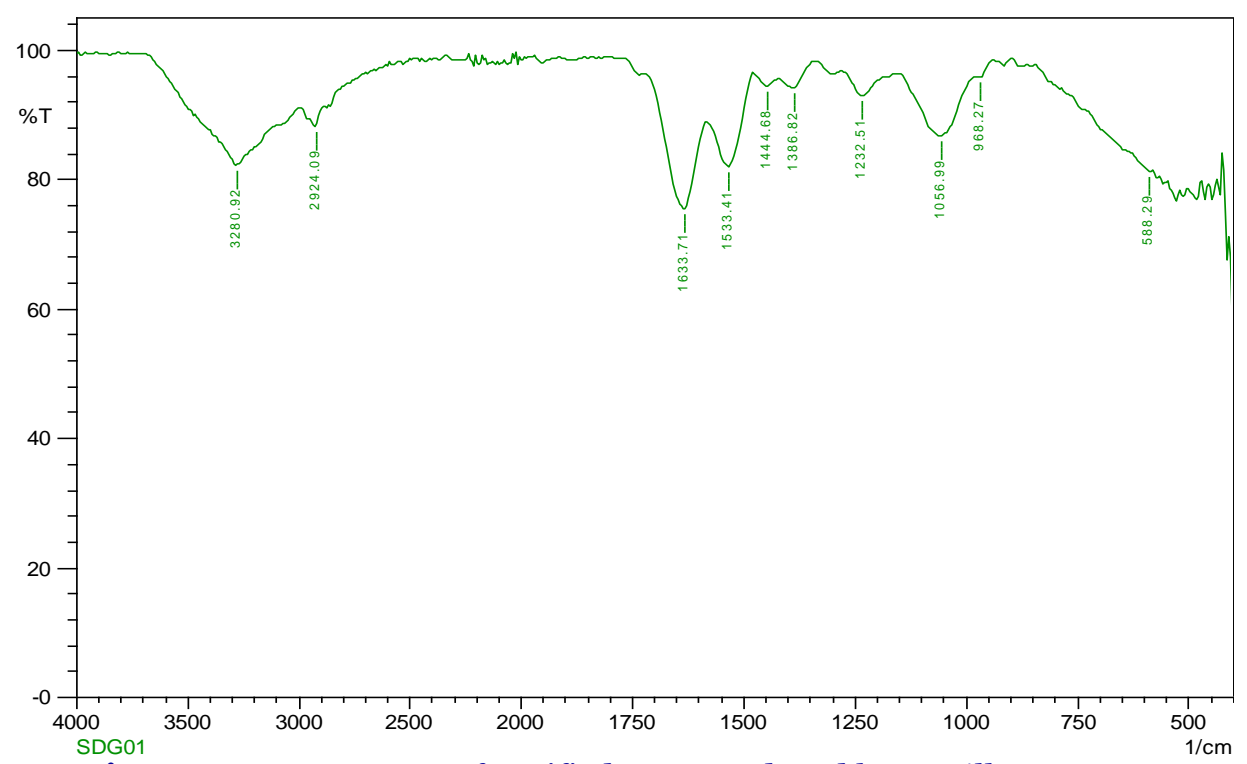

Fig. 5: FT-IR spectrum of purified PHB produced by Bacillus cereus.

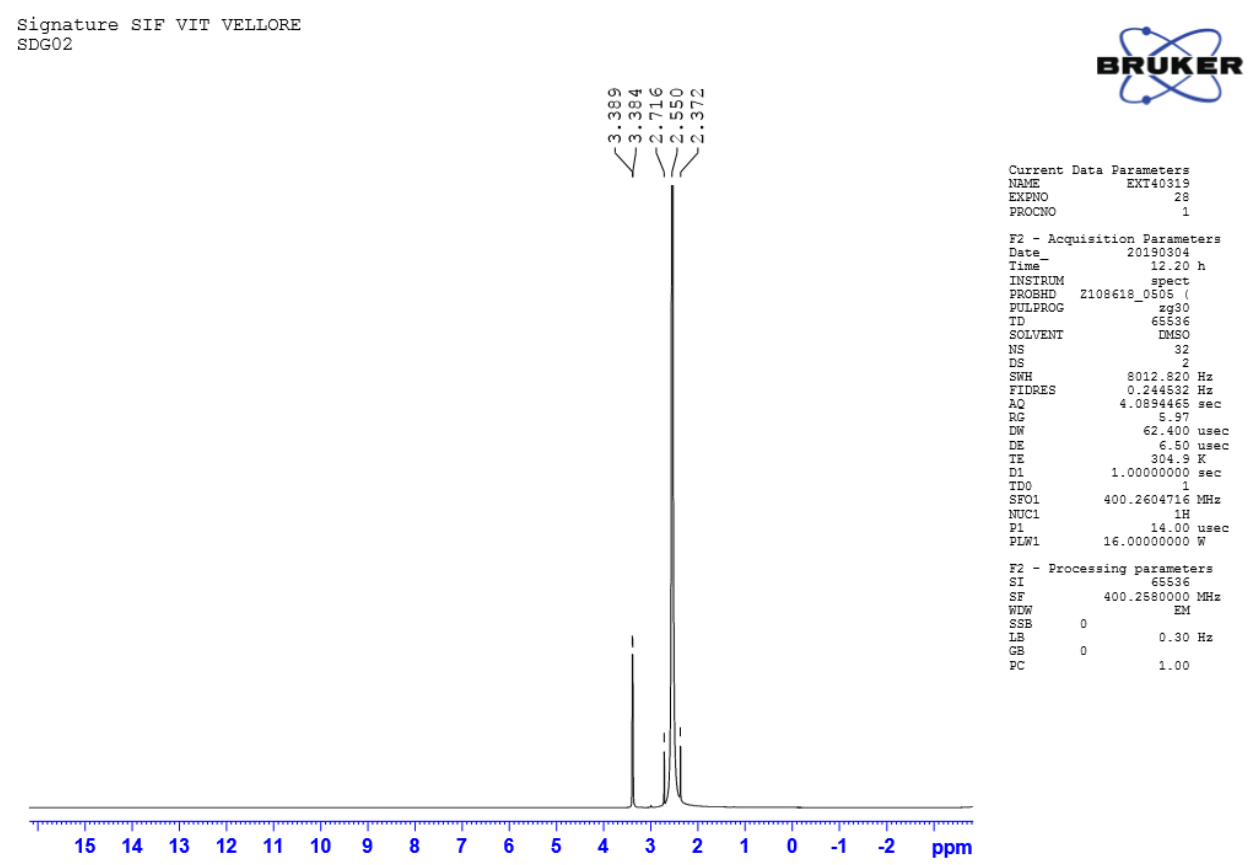

Fig. 6: ${ }^{1} \mathrm{H}$ NMR signals of PHB produced by Bacillus cereus.

The present study concludes that Bacillus cereus has got enormous enzymatic/metabolic potential to utilize glucose sources for growth and PHB production. Presence of glucose supported maximum PHB production by $B$. cereus Polymer got remarkable characters as per industrial viewpoint. The chemical nature of extracted polymers from Bacillus cereus was confirmed by ${ }^{1} \mathrm{H}-\mathrm{NMR}$ spectroscopy. The ${ }^{1} \mathrm{H}-\mathrm{NMR}$ spectra (Fig. 6) showed the presence of three signals, characteristic of the polymer of PHB. The ${ }^{1} \mathrm{H}-\mathrm{NMR}$ spectra of polymer extracted from isolate showed a doublets at $2.372 \mathrm{ppm}$, corresponding to the methyl group ($\mathrm{CH}_{3}$ ), and two multiplets at 2.55 and $3.389 \mathrm{ppm}$ corresponding to methylene group $(-\mathrm{CH} 2-)$ and methyne (-CH-) group, respectively. In this study with reference PHB standard, the nature of polymer produced by the isolate was confirmed. 


\section{Conclusion}

The Bacillus cereus isolated from sludge soil of Hosur lake area was able to efficiently utilize the glucose as nutrient source for PHB production. The first line of impression of this study concludes that the glucose could directly serve as an inexpensive nutrient source for production of biodegradable plastic. Thus, this study may solve the problem of high production cost of biodegradable bioplastic and help in the conservation of petroleum products which were used in the commercial production of plastic production.

\section{Acknowledgement}

The authors would like to thank the PG and Research Centre in Biotechnology, M.G.R. College, Hosur, for providing sophisticated laboratory support to successfully complete this study.

\section{Conflict of interest statement}

Authors declare that they have no conflict of interest.

\section{References}

Arshad, M.U., Jamil, N., Naheed, N., Hasnain, S., 2007. Analysis of bacterial strains from contaminated and non-contaminated sites for the production of biopolymers. Afr. J. Biotechnol. 6, 1115-1121.

Batcha, A.F.M., Prasad, D.M.R., Khan, M.R., Abdullah, H., 2014. Biosynthesis of poly (3hydroxybutyrate) (PHB) by Cupriavidus necator H16 from Jatropha oil as carbon source. Biopro. Biosy. Eng. 37, 943-951.

British Plastics Federation, 2017. Oil Consumption URL

http://www.bpf.co.uk/Press/Oil Consumptio n.aspx (accessed 4.10.17).

Chee, J.Y., Yoga, S.S., Lau, N.-S., Ling, S.C., Abed, R.M.M. and Sudesh, K., (2015). Bacterially produced Polyhydroxyalkanoate (PHA): converting renewable resources into bioplastics. Microbial Biotech. 2, 1395-1404.

Das, R., Pal, A., Paul, A. K., 2017. Production of biopolyester poly (3-hydroxybutyrate) by Bacillus cereus RCL 02, a leaf endophyte of Ricinus communis L. J. Microbiol. Biotechnol. Res. 7(4), 32-41.
Full, T.D., Jung, D.O., Madigan, M.T., 2006. Production of poly- $\beta$-hydroxyalkanoates from soy molasses oligosaccharides by new, rapidly growing Bacillus species. Lett. Appl. Microbiol. 43, 377-384.

Hori, K., Kaneko, M., Tanji, Y., Xing, X-H., Unno H., 2002. Construction of self-disruptive Bacillus megaterium in response to substrate exhaustion for polyhydroxybutyrate production. Appl. Microbiol. Biotechnol. 59, 211-216.

Khanna, S., Srivastava, A. K., 2005. Statistical media optimization studies for growth and PHB production by Ralstonia eutropha. Proc. Biochem. 40(6), 2173-2182.

Liau, C.P., Ahmad, M. B., Shameli, K., Yunus, W. M. Z.W., Ibrahim, N. A., Zainuddin, N., Then, Y.Y., 2014. Preparation and characterization of polyhydroxybutyrate/polycaprolactone nanocomposites. Scient. World J. Volume 2014, Article ID 572726, 9 pages.

Madison, L.L., Huisman, G.W., 1999. Metabolic engineering of poly (3-hydroxyalkanoates): from DNA to plastic. Microbiol. Mol. Biol. Rev. 63, 21-53.

Marshall, C.W., LaBelle, E. V., May, H.D., 2013. Production of fuels and chemicals from waste by microbiomes. Curr. Opin. Biotech. 24, 391397.

Mudgal, S., Shailendra, L., Lorcan, K., Philippe, G.T., 2013. Analysis of the public consultation on the green paper European Strategy on Plastic Waste in the Environment. pp.1-91.

Panda, A. K., Singh, R. K., Mishra, D. K., 2010. Thermolysis of waste plastics to liquid fuel: A suitable method for plastic waste management and manufacture of value added products-A world prospective. Renew. Sustain. Energy Rev. 14, 233-248.

Panda, I., Balabantaray, S., Sahoo, S. K., Patra, N., 2018. Mathematical model of growth and polyhydroxybutyrate production using microbial fermentation of Bacillus subtilis. J. Chem. Eng. Commu. 205, 249-256.

Ramadas, N.V., Singh, S.K., Soccol, C.R., Pande, A., 2009. Polyhydroxybutyrate production using agroindustrial residue as substrate by Bacillus sphaericus NCIM 5149. Braz. Arch. Biol. Technol. 52, 17-23.

Schlegal, H., Gottashalk, G., Bartha, R. V., 1961. Formation and utilization of polyhydroxyl butyric acid by Knallgas bacteria 
(Hydrigenomas). Nature (Lond.). 191, 463465.

Shivakumar, S., 2012. Polyhydroxybutyrate (PHB) production using agro-industrial residue as substrate by Bacillus thuringiensis IAM 12077 . Int. J. Chem. Tech. Res. 4, 1158-1162.

Singh, G., Mittal, A., Kumari, A., Goel, V., Aggarwal, N.K., Yadav, A., 2011. Optimization of poly- $\beta$-Hydroxybutyrate production from Bacillus species. Eur. J. Biol. Sci. 3, 112-116.

Thammasittirong, A., Saechow, S., Na-Ranong Thammasittirong, S., 2017. Efficient poly hydroxybutyrate production from Bacillus thuringiensis using sugarcane juice substrate. Turk. J. Biol. 41, 992-1002.

Tripathi, A.D., Srivastava, S.K., Singh, R.P., 2013. Statistical optimization of physical process variables for bio-plastic (PHB) production by Alcaligenes sp. Biomass Bioenergy 55, 243-250.

Valappil, S.P., Misra, S.K., Boccaccini, A.R., Keshavarz, T., Bucke, C., Roy, I., 2007. Largescale production and efficient recovery of PHB with desirable material properties, from the newly characterized Bacillus cereus SPV. J. Biotechnol. 132, 251-258.

Van-Thuoc, D., Quillaguaman, J., Mamo, G., Mattiasson, B., 2008. Utilization of agricultural residues for poly (3-hydroxybutyrate) production by Halomonas boliviensis LC1. J. Appl. Microbiol. 104, 420-428.

Yezza, A., Fournier, D., Halasz, A., Hawari, J., 2006. Production of PHA from methanol by a new methylotropic bacterium Methlobacterium sp.GW2. Appl. Microbiol. Biotech. 73, 211-218.

\section{How to cite this article:}

Murali, P., Mamatha, K., Mathiyazhagan, N., 2019. Screening of PHB biopolymer producing Bacillus cereus from municipal sludge waste. Int. J. Curr. Res. Biosci. Plant Biol. 6(8), 13-20.

doi: https://doi.org/10.20546/ijcrbp.2019.608.003 